\title{
Implausibility of the vibrational theory of olfaction
}

\author{
Eric Block ${ }^{a, 1}$, Seogjoo Jang ${ }^{b, 1}$, Hiroaki Matsunami ${ }^{c, 1}$, Sivakumar Sekharand, Bérénice Dethier ${ }^{a}$, Mehmed Z. Ertem ${ }^{\text {d,e }}$, \\ Sivaji Gundalaa, Yi Pan ${ }^{f}$, Shengju Lif, Zhen Lif, Stephene N. Lodge ${ }^{a}$, Mehmet Ozbil ${ }^{d}$, Huihong Jiang ${ }^{f}$, Sonia F. Penalba ${ }^{a}$, \\ Victor S. Batista ${ }^{d}$, and Hanyi Zhuang ${ }^{f, g, 1}$
}

\begin{abstract}
${ }^{a}$ Department of Chemistry, University at Albany, State University of New York, Albany, NY 12222; bepartment of Chemistry and Biochemistry, Queens College, and Graduate Center, City University of New York, Flushing, NY 11367; 'Department of Molecular Genetics and Microbiology and Department of Neurobiology, Duke Institute for Brain Sciences, Duke University Medical Center, Durham, NC 27710; ${ }^{d}$ Department of Chemistry, Yale University,

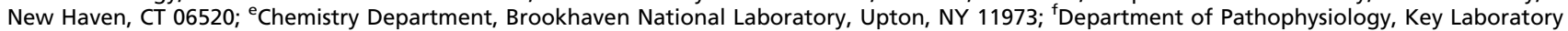
of Cell Differentiation and Apoptosis of Ministry of Education, Shanghai Jiaotong University School of Medicine, Shanghai 200025, China; and Institute of Health Sciences, Shanghai Jiao tong University School of Medicine/Shanghai Institutes for Biological Sciences of Chinese Academy of Sciences, Shanghai 200031, China
\end{abstract}

Edited by Jerrold Meinwald, Cornell University, Ithaca, NY, and approved March 31, 2015 (received for review February 20, 2015)

The vibrational theory of olfaction assumes that electron transfer occurs across odorants at the active sites of odorant receptors (ORs), serving as a sensitive measure of odorant vibrational frequencies, ultimately leading to olfactory perception. A previous study reported that human subjects differentiated hydrogen/ deuterium isotopomers (isomers with isotopic atoms) of the musk compound cyclopentadecanone as evidence supporting the theory. Here, we find no evidence for such differentiation at the molecular level. In fact, we find that the human musk-recognizing receptor, OR5AN1, identified using a heterologous OR expression system and robustly responding to cyclopentadecanone and muscone, fails to distinguish isotopomers of these compounds in vitro. Furthermore, the mouse (methylthio)methanethiol-recognizing receptor, MOR244-3, as well as other selected human and mouse ORs, responded similarly to normal, deuterated, and ${ }^{13} \mathrm{C}$ isotopomers of their respective ligands, paralleling our results with the musk receptor OR5AN1. These findings suggest that the proposed vibration theory does not apply to the human musk receptor OR5AN1, mouse thiol receptor MOR244-3, or other ORs examined. Also, contrary to the vibration theory predictions, muscone- $d_{30}$ lacks the $1,380-$ to $1,550-\mathrm{cm}^{-1}$ IR bands claimed to be essential for musk odor. Furthermore, our theoretical analysis shows that the proposed electron transfer mechanism of the vibrational frequencies of odorants could be easily suppressed by quantum effects of nonodorant molecular vibrational modes. These and other concerns about electron transfer at ORs, together with our extensive experimental data, argue against the plausibility of the vibration theory.

olfaction | isotopomers | cyclopentadecanone | muscone | electron transfer

n 1870, the British physician William Ogle wrote: "As in the eye and the ear the sensory impression is known to result not from the contact of material particles given off by the object seen or heard, but from waves or undulations of the ether or the air, one cannot but suspect that the same may be true in the remaining sense, and that the undulatory theory of smell... [may be] the true one" $(1,2)$. Of the 29 different "theories of odour" listed in the 1967 edition of The Chemical Senses (3), nine associate odor with vibrations, particularly those theories championed by Dyson $(4,5)$ and Wright (6-8). However, the premise that olfaction involves detection of vibrational frequencies of odorants remains highly speculative because neither the structures of the odorant receptors (ORs) nor the binding sites or the activation mechanisms triggered upon odorant binding to ORs have been established. In 1996-1997, Turin (9-12) elaborated on the undulatory theory of smell, as considered in more detail below, and suggested that a mechanism analogous to inelastic electron tunneling spectroscopy (13) may be involved, where tunneling electrons in the receptor probe the vibrational frequencies of odorants. In 2013, Gane et al. (14) commented that "whether olfaction recognizes odorants by their shape, their molecular vibrations, or both remains an open and controversial question" and that "a convenient way to address [this question] is to test for odor character differences between deuterated and nondeuterated odorant isotopomers since these have identical ground-state conformations but different vibrational modes." Gane et al. (14) also stated that a particularly appropriate test case would involve odorants containing "more $\mathrm{CH}$ group.. [such as] musks [which] are among the largest odorants and typically contain 15-18 carbons and 28 or more hydrogens."

In judging the plausibility of the vibration theory, we use a multipronged approach:

i) We consider the concepts of shape vs. vibration theory and odorant perception vs. reception.

ii) As a test of the vibration theory, we have prepared a series of isotopomers of musks and other compounds, containing up to $30 \mathrm{C}-\mathrm{H}$ or $\mathrm{C}-\mathrm{D}$ bonds as test odorants, which are evaluated using in vitro activation of receptors identified by us and other groups as being highly responsive to these isotopomers.

iii) We consider the confounding effects of impurities and isotope effects in interpreting odorant perception, as well as the validity of requirements for specific IR bands for recognition of musks by their receptors.

\section{Significance}

The vibrational theory of olfaction posits detection of odorants through their vibrational frequencies rather than solely through "hand-in-glove" substrate/enzyme-like odorant-odorant receptor (OR) interactions. To test the theory, we compare responses of different human and mouse ORs toward deuterated and undeuterated isotopomers (isotopic atom isomers) of receptorresponsive odorants because isotopomers should differ in their molecular vibrational frequencies. However, no differences in receptor response are seen with any tested labeled/unlabeled odorant/receptor pairs. Because published behavioral studies have shown that humans can distinguish isotopomers, perireceptor events or impurities, rather than receptor-level vibrational effects, are suggested. Because theoretical aspects of the vibration theory are also found wanting, the vibration theory is deemed implausible in the absence of compelling receptor-level experimental evidence to the contrary.

Author contributions: E.B., S.J., H.M., V.S.B., and H.Z. designed research; E.B., S.J., H.M., S.S. B.D., M.Z.E., S.G., Y.P., S.L., Z.L., S.N.L., M.O., H.J., S.F.P., V.S.B., and H.Z. performed research E.B. contributed new reagents/analytic tools; E.B., S.J., H.M., S.S., B.D., M.Z.E., S.G., Y.P., S.N.L. M.O., S.F.P., V.S.B., and H.Z. analyzed data; and E.B., S.J., H.M., V.S.B., and H.Z. wrote the paper The authors declare no conflict of interest.

This article is a PNAS Direct Submission.

Freely available online through the PNAS open access option.

See Commentary on page 6525 .

${ }^{1}$ To whom correspondence may be addressed. Email: eblock@albany.edu, seogjoo.jang@ qc.cuny.edu, hiroaki.matsunami@duke.edu, or hanyizhuang@sjtu.edu.cn.

This article contains supporting information online at www.pnas.org/lookup/suppl/doi:10. 1073/pnas.1503054112/-/DCSupplemental. 
iv) We examine the physical validity of the models developed to support the vibration theory.

v) We consider the specific limitations of our in vitro approach using isotopomers to evaluate the vibration theory, based primarily on results obtained with a single identified human musk OR, in addition to other OR/ligand pairs.

vi) We consider plausible nonvibration theory models for docking of musks to the human musk receptor, OR5AN1, where the musk carbonyl group functions as a hydrogen bond acceptor.

Gane et al. (14) have framed the argument for olfactory discrimination of hydrogen isotopomers as one of "shape" vs. "vibration." However, neither the binding modes of isotopomers nor their activation mechanisms are known. ORs belong to the superfamily of class A G protein-coupled receptors (GPCRs), which are known to be activated through allosteric conformational changes induced upon ligand binding even without triggering any kind of electron transfer processes. Ligand-receptor interactions can be both attractive and repulsive, involving hydrogen bonding, van der Waals, cation $-\pi, \pi-\pi$, ion-ion, dipoledipole, steric, and hydrophobic interactions with the receptor, with water channels and bridging water molecules mediating hydrogen bonds, as well as metal-ion coordination, as we have recently demonstrated in the latter case $(15,16)$. Therefore, molecular shape can be considered a "straw-man" alternative to the vibration theory when describing the differing affinities of ligands bound to GPCRs $(17,18)$, including isotopomers (19, 20). Some of these attractive and repulsive interactions were identified in 1940 by Pauling and Delbrück (21), who note that interacting biomolecules "must have complementary surfaces, like die and coin, and also a complementary distribution of active groups." In addition, shape-related features are misrepresented by vibration theory proponents. For example, Franco et al. (17) stated: "Given that proteins are chiral, a shape-only theory cannot account for the identical odors of most enantiomeric pairs," echoing similar comments by Turin (22): "One would therefore generally expect enantiomers to have completely different smells. This is emphatically not the case." However, these assertions are clearly at odds with the highly developed ability of mice and other mammals to discriminate an array of nonpheromonal chiral odorant enantiomeric pairs (23-25), with the divergent in vitro responses to enantiomers by different combinations of ORs (26) and, in particular, with the highly selective response of the musk-sensitive mouse receptor, MOR215-1, to $(R)$-muscone ("l-muscone") compared with $(S)$-muscone ("d-muscone") (27).

In addition to our concerns regarding shape, a second issue relates to describing how different smells are perceived, that is, the perception of an odorant. It is known that in vivo perception of odorants reflects the totality of perireceptor events as well as odorant-OR interactions (reception). Volatile odorants enter the nasal passage, where they dissolve in the nasal mucus overlying the olfactory epithelium and are then rapidly detected by ORs on the cilia of the olfactory sensory neurons, ultimately leading to signaling $(28,29)$. It is the mechanism of odorant-OR interactions, the reception of the odorant, that we seek to examine with isotopomers to determine whether the vibration theory is plausible, displaying isotope effects, because perception could be influenced by isotope effects due to the perireceptor events involving mucosal components, such as enzymes, mucopolysaccharides, salts, and antibodies.

Whether deuterated and nondeuterated odorant isotopomers can be distinguished by smell and, even if they can, whether this distinction validates the vibration theory is a matter of contention. A 2001 paper by Haffenden et al. (30) reported that benzaldehyde- $\mathrm{d}_{6}$ gave a statistically significant difference in odor perception relative to normal benzaldehyde, in support of the vibration theory. However, this study has been criticized for lacking double-blind controls to eliminate bias and because it used an anomalous version of the duo-trio test (31). Furthermore, the study failed to account for perireceptor events, namely, the enzyme-mediated conversion of odorants that has been shown to occur in nasal mucus. For example, benzaldehyde is converted to benzoic acid (32), a reaction potentially subject to significant primary isotope effects $(2,33,34)$, which could explain the difference in odor perception for the benzaldehyde isotopomers. Earlier claims that human subjects can distinguish odors of acetophenone isotopomers $(9,35)$ have been shown to be untrue $(14,31)$. Recent studies indicate that Drosophila melanogaster can distinguish acetophenone isotopomers $(36,37)$ and that Apis mellifera L., the honey bee, can be trained to discriminate pairs of isotopomers (38). These studies differ from earlier insect studies in which isotopomer discrimination was not found. For example, systematic deuteration of 4-( $p$-hydroxyphenyl)-2-butanone acetate, a Dacus cucurbitae Coquillett (the male melon fly) attractant, did not affect the attractiveness of the compound to the fly (39); deuteration of alarm pheromones failed to alter the response toward these compounds by Pogonomyrmex badius worker ants (40); and honey bees could not distinguish between deuterated and nondeuterated nitrobenzene (41).

Concerns have been raised (42) about aspects of the Drosophila study (36), which is "behavioural and not at the receptor level" (2) (also a concern with the Apis study). Also, given that the ORs and their downstream signaling in Drosophila and humans are completely unrelated, the Drosophila study should not be considered predictive of the ability of humans to distinguish isotopomers $(2,17)$. In view of the above discussion, it is interesting that in a blinded behavioral study, smell panelists distinguished between deuterated and nondeuterated isotopomers of cyclopentadecanone (1; Fig. $1 A$ ) and other musk odorants (14).

Here, we study the response of human musk-sensitive OR5AN1, identified through screening of heterologously expressed human ORs, to cyclopentadecanone (1) and muscone (4) isotopomers. We also present pharmacological data on the response of mouse ORs to deuterated and nondeuterated acetophenone and benzaldehyde, as well as selected ${ }^{13} \mathrm{C}$ isotopomers. In addition, we present related studies on the response of various human and mouse ORs to other deuterated and nondeuterated odorants, including (methylthio)methanethiol (MTMT, 8; Fig. 1C) and bis(methylthiomethyl) disulfide (9), studied in connection with our investigation of the role of copper coordination in the recognition of both sulfur-containing odorants by the mouse (methylthio)methanethiol receptor, MOR244-3 $(15,16)$. Insofar as the ability to distinguish odors of isotopomers directly tests the predictions of the vibration theory, the comparative response of human and mouse ORs to isotopomers of these selected ligands in the heterologous OR expression system constitutes a robust test of the vibration theory. Finally, we discuss the basis for recent vibration theories of olfaction and supporting computational evidence $(37,43-47)$ in light of well-established electron transfer theories (48). We point out that key assumptions underlying the vibration theory lack experimental support and are missing important physical features expected for biological systems.

\section{Experimental Results}

Response of a Human Musk OR to Deuterated and Nondeuterated Muscone and Other Musk Compounds. Because human subjects are reported to discriminate between fully deuterated and nondeuterated cyclopentadecanone (1; Exaltone) (14), we sought to perform a corresponding receptor activation assay in vitro.

First, from a commercial sample of cyclopentadecanone (1, Fig. $1 A$ ), we synthesized $\mathbf{1}-\mathrm{d}_{28}$ (fully deuterated), $\mathbf{1}-\mathrm{d}_{24}$, and $\mathbf{1}-\mathrm{d}_{4}$. Treatment of 1 (twice) with $\mathrm{Rh} / \mathrm{C}$ in $\mathrm{D}_{2} \mathrm{O}$ at $150{ }^{\circ} \mathrm{C}$ (49) gave cyclopentadecanol- $\mathrm{d}_{29}\left(2-\mathrm{d}_{29}\right)$, which was oxidized with $\mathrm{K}_{2} \mathrm{Cr}_{2} \mathrm{O}_{7} /$ acetone (50) and repeatedly recrystallized to give $1-\mathrm{d}_{28}$. Deuterium 
A
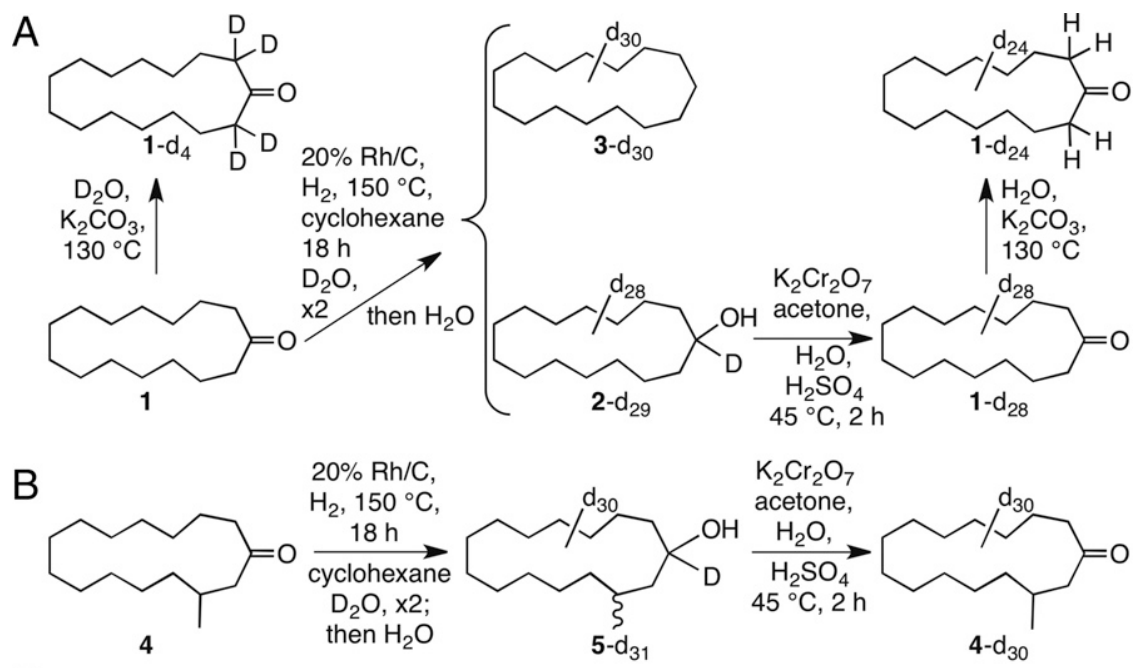

C $\mathrm{CD}_{3} \mathrm{~S}(\mathrm{O}) \mathrm{CD}_{3} \frac{\text { 1) } \mathrm{SOCl}_{2}, \mathrm{CH}_{2} \mathrm{Cl}_{2}}{\text { 2) }\left(\mathrm{H}_{2} \mathrm{~N}\right)_{2} \mathrm{C}=\mathrm{S}, \mathrm{Me}_{2} \mathrm{CO}}$<smiles>CO[AsH3]</smiles>
$\mathrm{I}_{2}, \mathrm{Et}_{3} \mathrm{~N}$

3) $\mathrm{NaOH}$

8- $\mathrm{d}_{5}$

Fig. 1. (A) Preparation of deuterated 1-3. Deuterium could be selectively introduced, or selectively removed, adjacent to the carbonyl group using $\mathrm{D}_{2} \mathrm{O} / \mathrm{K}_{2} \mathrm{CO}_{3}$ or $\mathrm{H}_{2} \mathrm{O} / \mathrm{K}_{2} \mathrm{CO}_{3}$, respectively, at $130{ }^{\circ} \mathrm{C}$; global replacement of all hydrogens could be achieved with $\mathrm{Rh} / \mathrm{C}$ in $\mathrm{D}_{2} \mathrm{O}$ at $150{ }^{\circ} \mathrm{C}$. Repetition led to more complete deuteration as well as reduction of 1 to $\mathbf{3}$ and 2; oxidation of $\mathbf{2}$ gave $\mathbf{1}$ with $\sim 98 \%$ deuteration. Chromatography of deuterated $\mathbf{1}$ with freshly distilled pentane followed by repeated recrystallization from methanol/water to constant melting point gave samples showing no new peaks in their ${ }^{1} \mathrm{H}$ NMR spectra, other than very weak peaks corresponding to those peaks seen in undeuterated 1 . (B) Deuterated $(97 \%)$ muscone 4 was prepared via alcohol 5 as above. (C) 8- $d_{5}$ and 2,4,5,7-tetrathiaoctane- $d_{10},\left(9-d_{10} ; 98 \%\right.$ deuterium) were prepared as shown. Details of these syntheses are provided in SI Appendix.

was selectively introduced into $\mathbf{1}$, or selectively removed from $1-\mathrm{d}_{28}$, adjacent to the carbonyl group using $\mathrm{D}_{2} \mathrm{O} / \mathrm{K}_{2} \mathrm{CO}_{3}$ or $\mathrm{H}_{2} \mathrm{O} / \mathrm{K}_{2} \mathrm{CO}_{3}$ (51), giving 1- $\mathrm{d}_{4}$ and $1-\mathrm{d}_{24}$, respectively, at $130{ }^{\circ} \mathrm{C}$. A sham sample of $\mathbf{1}$, which underwent all of the same procedures as 1- $\mathrm{d}_{28}$, but with $\mathrm{H}_{2} \mathrm{O}$ instead of $\mathrm{D}_{2} \mathrm{O}$, was also included as a negative control. Both $\mathbf{2} / \mathbf{2}-\mathrm{d}_{29}$ and $\mathbf{3}-\mathrm{d}_{30}$, a byproduct in the catalytic reduction of $\mathbf{1}$, and nondeuterated $\mathbf{3}$ were also tested for receptor activation. Similarly, $(R, S)$-muscone $[4 ;(R, S)$-3-methylcyclopentadecanone] was converted to $4-\mathrm{d}_{30}$ by way of cis/trans-3-methylcyclopentadecanol- $\mathrm{d}_{31}$ $\left(5-\mathrm{d}_{31}\right)$ (Fig. $\left.1 B\right)$. Compounds 4 and $4-\mathrm{d}_{30}$ are baseline-resolved by GC and show very different IR spectra (Fig. 2; experimental details of synthesis and characterization of deuterated compounds 1-5 are provided in SI Appendix). Contrary to statements by Gane et al. (14) that a musk receptor "detects vibrations in the $1,380-1,550 \mathrm{~cm}^{-1}$ range," and that musk odor requires that "the molecule has intense bands in that region," the IR spectrum of $4-\mathrm{d}_{30}$ is devoid of 1,380 - to 1,550- $\mathrm{cm}^{-1}$ absorption (Fig. 2).

Second, using a heterologous OR expression system $(52,53)$, we performed parallel screenings of all deuterated and nondeuterated versions of $\mathbf{1}$ on the human OR repertoire. Among all 330 human ORs screened, we identified one OR, OR5AN1, that is a bona fide receptor for $\mathbf{1}$ and its isotopomers (SI Appendix, Fig. S3.1). OR5AN1 also responds strongly to other related musk analogs, including muscone, cyclopentadecanol, and $\omega$-pentadecalactone (Exatolide) (SI Appendix, Fig. S3.3). This response is consistent with a recent report (27), in which OR5AN1 was identified as a human muscone OR, based on homology to the mouse OR MOR215-1, functionally cloned from muscone-responsive glomeruli; a second report on OR5AN1 as the only functional human homolog of mouse muscone ORs in vivo (54); and a third report that only a small number of receptors are thought to be involved in sensing musk odor (55). Our screening and the following confirmation experiments did not reveal any human OR that responded to only one, two, or three of the four isotopomers of $\mathbf{1}$.

Third, we tested whether or not OR5AN1 responded similarly to isotopomers of the different musk analogs. We found that all four different isotopomers of $\mathbf{1}$ gave highly similar responses and the $\mathrm{EC}_{50}$ values of the respective dose-response curves were not significantly different (Fig. 3A, Left, and SI Appendix, Table S3.2A). In addition, we tested $\mathbf{2}$ alongside fully $\mathrm{C}-\mathrm{D}$ deuterated isotopomer $2-\mathrm{d}_{29}$ and found that even though this compound evoked a much smaller response, similar response levels were seen between the deuterated and nondeuterated versions of this compound ( $S I \mathrm{Ap}$ pendix, Fig. S3.2B and Table S3.2B). We also found the hydrocarbon analogs cyclopentadecane $(\mathbf{3})$ and $\mathbf{3}-\mathrm{d}_{30}$ to be inactive (SI Appendix, Fig. S3.2B). We also tested whether or not OR5AN1 responded similarly to isotopomers of $(R, S)$-muscone (4). Again, we found similar responses between the undeuterated $(R, S)$ muscone and its fully deuterated $\mathrm{d}_{30}$ isotopomer $\left(4-\mathrm{d}_{30}\right)$ (Fig. $3 A$, Right, and SI Appendix, Table S3.2A).

Mouse ORs for Acetophenone and Benzaldehyde Show Similar Responses to All Isotopomers Tested. We assayed isotopomers of acetophenone (6) and benzaldehyde (7) in our system using cognate mouse ORs. Similar to the case of cyclopentadecanone, no significant difference was seen between nondeuterated and deuterated versions for all of the ORs tested (Fig. $4 A$ and $B$ and $S I$ Appendix, Table S3.2A). In addition, ${ }^{13} \mathrm{C}$-labeled isotopomers may present a good test for validating/invalidating the vibration theory because they do not significantly alter vibrational frequencies (42). We also included $6-\alpha, \beta-{ }^{13} \mathrm{C}_{2}$ and $6-{ }^{13} \mathrm{C}_{8}$ as well as ${ }^{13} \mathrm{CHO}-7-{ }^{13} \mathrm{C}_{1}$ to test against their ${ }^{12} \mathrm{C}$ counterparts in our odorant panel. We again found no significant differences among all isotopomers tested (Fig. $4 A$ and $B$ and SI Appendix, Table S3.2A), indicating that neither vibrational frequencies nor other factors, such as association/dissociation rates, are contributing to the level of OR activation.

Additional ORs Respond Similarly to Isotopomeric Ligands in Heterologous Cells. We investigated whether other ORs respond differently to isotopomer pairs by assaying 14 other known receptor/ ligand pairs using 10 human and mouse ORs and 10 odorous 


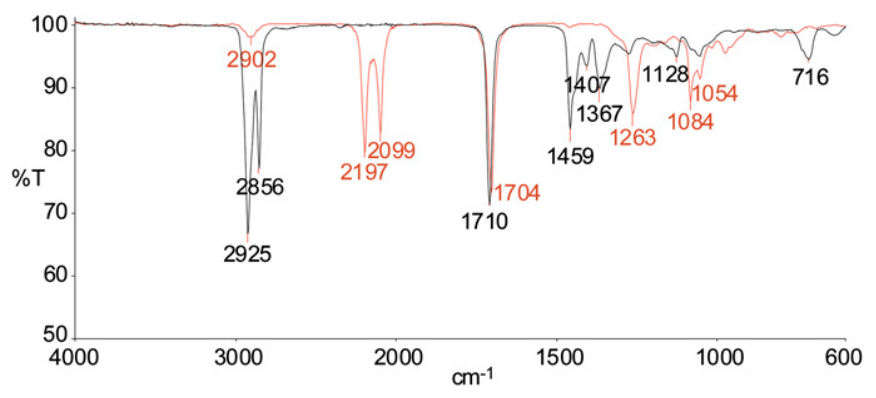

Fig. 2. Superimposed IR spectra of $4-d_{30}$ (red trace) and undeuterated muscone (4; black trace) showing that $4-d_{30}$ is devoid of IR absorption in the 1,380 - to $1,550-\mathrm{cm}^{-1}$ region.

ligands with purchased or synthesized H/D isotopomers, including octanol and octanol- $\mathrm{d}_{17}$, discriminated by Drosophila in the aforementioned study (36). Our assay included MTMT (8) and MTMT$\mathrm{d}_{5}\left(\mathbf{8}-\mathrm{d}_{5}\right)$ (Fig. $\left.1 C\right)$, as well as bis(methylthiomethyl) disulfide (9) and 9- $\mathrm{d}_{10}$, ligands for mouse receptor MOR244-3, which are notable for requiring copper for ligand binding and whose active site we have modeled $(15,16)$. Deuterated compound $\left(8-\mathrm{d}_{5}\right)$ was prepared in several steps from dimethyl sulfoxide- $\mathrm{d}_{6}$ and then oxidized to $9-\mathrm{d}_{10}$; these compounds had $\sim 100 \% \mathrm{~d}_{5}$ and $\mathrm{d}_{10}$ deuterium incorporation, respectively, according to GC-MS. When 8 and 9 were tested in the presence of $30 \mu \mathrm{M} \mathrm{Cu}^{2+}$, no differential receptor activity was seen, which was also the case for the other isotopomeric pairs tested (Fig. $3 B$ and SI Appendix, Fig. S3.2B and Table S3.2 $A$ and $B$ ).

Concerns Involving Impurities. When comparing odors of isotopomers, it is essential to ensure that what is being measured is the odor of pure isotopomers devoid of impurities, because trace impurities could lead to a differential response at the organism or receptor level. For example, despite Turin's claims of different odors for deuterated and nondeuterated dimethyl sulfide (35), no differences were seen in the OR response to dimethyl sulfide (10) and 10- $\mathrm{d}_{6}$ (Fig. $3 B$ and SI Appendix, Table S3.2A). These results are consistent with reports that the odor of commercial samples of dimethyl sulfide is due to impurities, which can be removed by washing with aqueous $\mathrm{HgCl}_{2}$ (56). We suggest that commercial samples of $\mathbf{1 0}-\mathrm{d}_{6}$ are of much higher purity than samples of dimethyl sulfide; the former may have lower levels of these impurities. In general, this difference in purity is anticipated between undeuterated and deuterated isotopomers, based on the multistep procedures involved in isotopic labeling and expectations based on the much higher cost of the deuterated compounds.

In a more pertinent example, Gane et al. (14) report the ${ }^{1} \mathrm{H}$ NMR data for deuterated cyclopentadecanone (1), purified by silica gel chromatography using $9: 1$ hexane/ether, as " $\delta 2.37$ $(\mathrm{m}, 0.2 \mathrm{H}), 1.59(\mathrm{~m}, 0.22 \mathrm{H}), 1.30-1.20(\mathrm{~m}, 1.72 \mathrm{H}), 0.84-0.87$ $(\mathrm{m}, 0.25 \mathrm{H})[\mathrm{m}=$ multiplet; $\mathrm{nH}=$ relative number of protons found by NMR integration, e.g., $0.2 \mathrm{H}]$." Notably, the ${ }^{1} \mathrm{H}$ NMR spectrum of pure, commercial 1 (fig. 1 of ref. 14 and SI Appendix, Fig. S2.7) shows the highest field peak at $\delta 1.30-1.20$, with no evidence of absorption at $\delta$ 0.84-0.87, which leads us to question the assertion of Gane et al. (14) for their deuterated $\mathbf{1}$ that "No impurities are seen in the spectra." In our hands, the ${ }^{1} \mathrm{H}$ NMR spectra (SI Appendix, Fig. S2.6) for chromatographed and repeatedly recrystallized samples of $1-\mathrm{d}_{28}$ lack the unidentified impurity peak at $\delta 0.84-0.87$ in the deuterated 1 of Gane et al. (14), which was not seen in the ${ }^{1} \mathrm{H}$ NMR spectrum of their [or our (SI Appendix)] commercial 1. This impurity peak, seen in our deuterated samples when commercial, unpurified chromatography grade hexanes were used for chromatography, but not with redistilled pentane, could possibly have compromised the smell testing performed by Gane et al. (14), given that for odor evaluation in their study, "after silica gel purification, aliquots of the deuterated musks were diluted in ethanol and their odor character assessed on smelling strips," and that the $\delta 0.84-0.87$ impurity peak constituted $10 \%$ by integration of all residual proton signals. With regard to GC purification before additional smell testing, it is not known whether or not the compound(s)
A

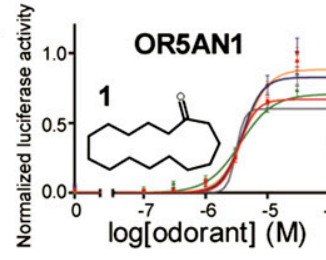

- 1

- $1-d_{4}$ $-5.46$

$-5.37$

- 1-d 24

$-5.41$

- 1-d 28

$-5.44$

- 1 SHAM -5.51

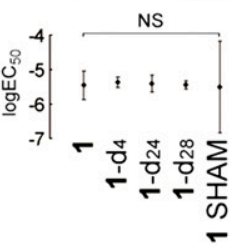

B

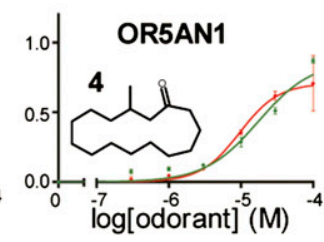

- $4 \quad-5.02$

- 4-d $30 \quad-4.76$

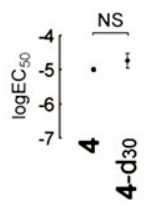

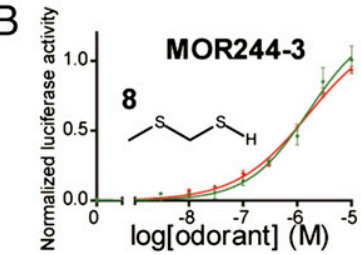

- 8

- 8-d5 $\quad-5.79$

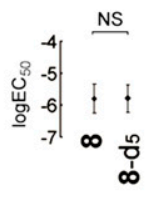

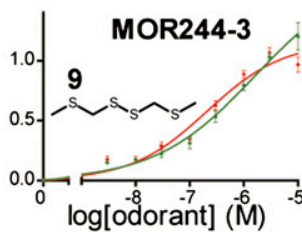

- 9

$-6.68$

- $9-d_{10}-5.80$

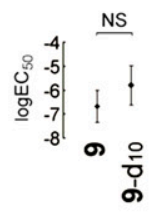

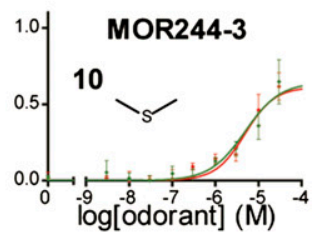

- $10 \quad-5.37$

- 10-d6 - -5.26

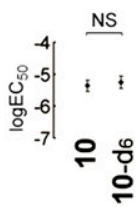

Fig. 3. Dose-response curves of OR5AN1 to isotopomers of cyclopentadecanone (1) and muscone (4) (A) and MOR244-3 to isotopomers of MTMT (8), bis(methylthiomethyl) disulfide (9), and dimethyl sulfide (10) (B). Best-fit logEC $\mathrm{C}_{50}$ values of the curves are shown alongside the graph legends (placed below the graphs). Scatter plots with $95 \%$ confidence interval $\log \mathrm{EC}_{50}$ values and indicating statistical significances between the logEC $\mathrm{C}_{50}$ values among isotopomers are also shown below the corresponding graphs. In $B, 30 \mu \mathrm{M}$ of copper was added upon odorant stimulation. "SHAM" indicates nondeuterated cyclopentadecanone subjected to the same chemical synthetic procedures as the deuterated samples without $\mathrm{D}_{2} \mathrm{O}$ addition. NS, not significant. For all dose-response curve graphs, the chemical structures of the respective compounds are shown within the graphs and normalized responses are shown as mean $\pm \operatorname{SEM}(n=3)$. 
A

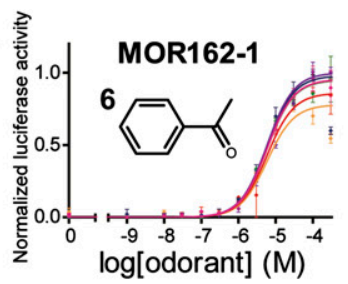

$-5.21$

$-5.40$

$-5.29$

$-5.27$

$-5.04$

$-5.21$
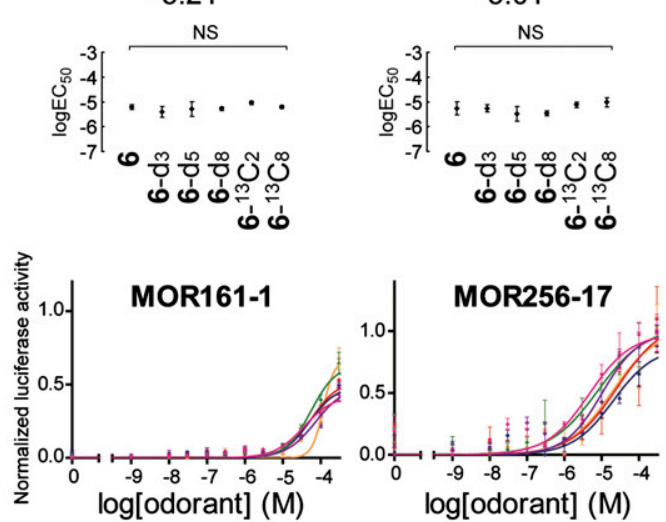

$-5.26$

$-5.26$

$-5.49$

$-5.47$

$-5.11$

$-5.01$

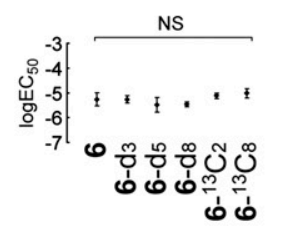

$-4.26$

$-3.94$

$-4.30$

$-4.26$

$-4.14$

$-4.44$

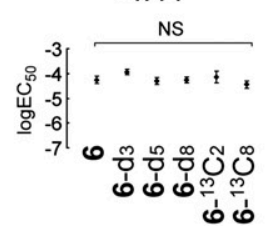

$-4.58 \cdot 6$

$-4.73 \cdot 6-\mathrm{d}_{3}$

$-4.69 \cdot 6-\mathrm{d} 5$

$-5.10 \cdot 6-d_{8}$

$-4.99 \cdot 6-{ }^{13} \mathrm{C}_{2}$

$-5.33 \cdot 6{ }^{-13} \mathrm{C}_{8}$

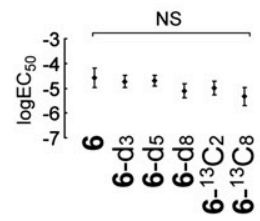

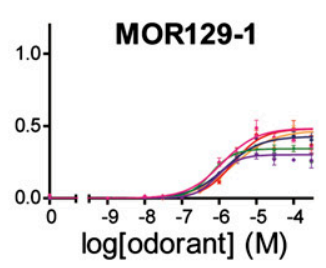
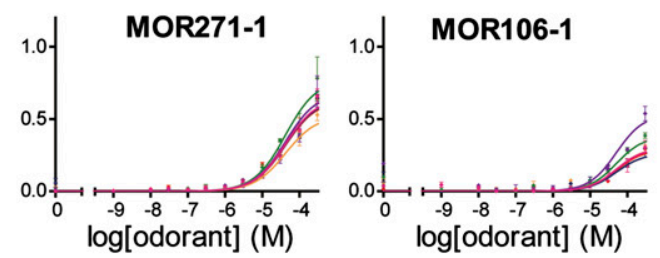

$-5.64$

$-5.60$

$-5.83$

$-6.22$

$-6.06$

$-5.92$

\section{$-4.49$}

$-4.47$

$-4.36$

$-4.40$

$-4.24$

$-4.35$
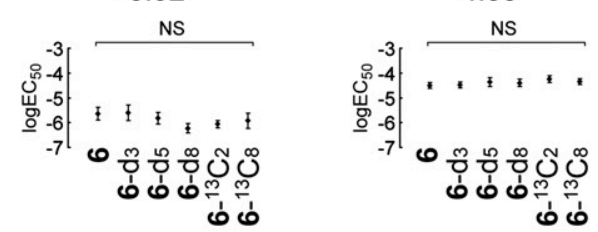

$\mathrm{B}$ 章
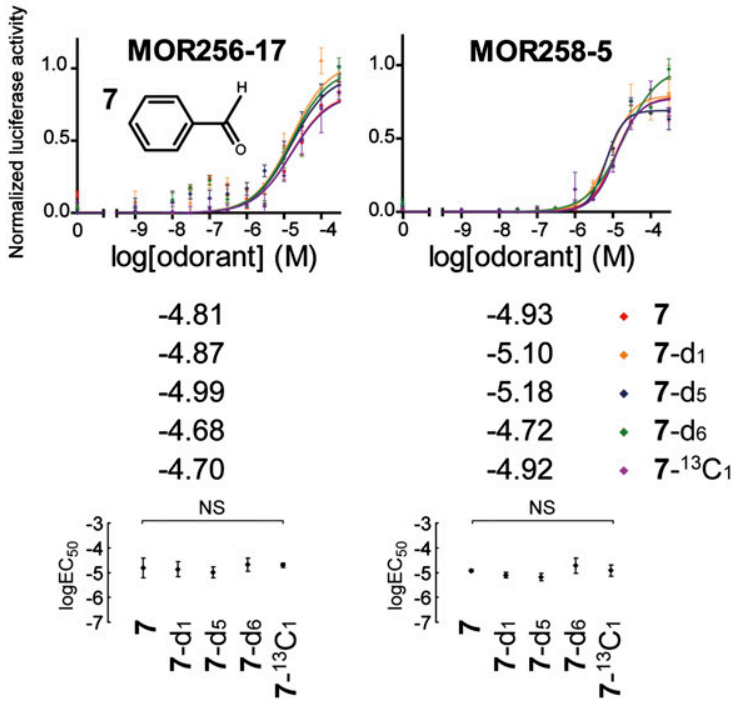

Fig. 4. Dose-response curves of various mouse receptors to isotopomers of acetophenone (6) $(A)$ and benzaldehyde (7) (B). Best-fit logEC 50 values of the curves are shown alongside the graph legends (placed below the graphs). Scatter plots with $95 \%$ confidence interval logEC $C_{50}$ values and indicating statistical significances between the $\log \mathrm{EC}_{50}$ values among isotopomers are also shown below the corresponding graphs.

responsible for the additional $\delta 0.84-0.87$ impurity peak, or decomposition products of the compound(s) in the hot injection port, coelute with deuterated musks.

Concerns Involving Isotope Effects. Although differences in perception of hydrogen/deuterium isotopomers have been invoked as evidence supporting the vibration theory, it is important to recognize that changing $\mathrm{H}$ to $\mathrm{D}$ not only changes vibration but also intermolecular interactions, due to the lowering of zero point energy of bonds to $\mathrm{D}$ compared with $\mathrm{H}$. Thus, the acidity of $\mathrm{D}_{2} \mathrm{O}$ and $\mathrm{H}_{2} \mathrm{O}$ are different, hydrogen bonding of $\mathrm{O}-\mathrm{H}$ and $\mathrm{O}-\mathrm{D}$ bonds are different, boiling points and freezing points are different, etc. In particular, the gas chromatographic retention times of isotopomeric pairs in the present study are significantly different in all cases examined.

The lack of isotope effects of isotopomers 1-8 when interacting with the corresponding receptors is not unexpected, given that $\mathrm{C}-\mathrm{H} / \mathrm{C}-\mathrm{D}$ bonds are not likely to be broken during docking with the receptor. Comparative isotopomer-receptor interactions can be probed computationally. In fact, we have reported a quantum mechanics/molecular mechanics (QM/MM) model for the mouse OR MOR244-3 in complex with the organosulfur odorant MTMT (8) (16). The proposed binding site consists of a copper ion coordinated to the thioether sulfur atom of MTMT as well as to the $\mathrm{N}, \mathrm{S}$, and $\mathrm{O}$ atoms of $\mathrm{H} 105, \mathrm{C} 109$, and N202 residues. The QM/ MM calculations indicate that both the deuterated odorant $\left(\mathbf{8}-\mathrm{d}_{5}\right)$ and nondeuterated odorant $(\mathbf{8})$ have similar binding affinities, and that no difference in response is predicted upon deuteration, consistent with the experimental observations (SI Appendix).

In addition, we point out that isotope effects in odorant response at the behavioral/organismal level are not necessarily evidence in favor of the vibration theory. Perireceptor events and/or psychophysical processes are known to be important in olfaction $(2,57)$ and may result in different olfactory percept of isotopomers. For example, it has been proposed that the nasopharyngeal mucus "behaves like a polar chromatographic column" (58), with differential diffusion rates, air/mucus partition coefficients (59), and solubility toward dissolved odorants (60), potentially leading to separation of isotopomers. Because $\mathbf{1}$ and $\mathbf{1}-\mathrm{d}_{28}$ 
are separated by several minutes on a gas chromatographic column (14), and HPLC separation of H/D isotopomers is well known (61), isotopomer fractionation could contribute to perceived differences.

Furthermore, as noted by Brookes et al. (46), biotransformation enzymes reside within the mucus layer and "comparisons of odors could well be affected even by small differences in metabolism, for instance from reaction rates depending on isotope[s]." Because the Baeyer-Villager (B-V) reaction is known to be mediated (62) by oxidative enzymes (e.g., cytochrome P450), which are present in the nasopharyngeal mucus layer $(2,32,63,64)$, and the B-V reaction of deuterated cyclic ketones forming deuterated lactones is known to show an isotope effect (62), such a reaction might affect odor perception of pairs such as $\mathbf{1}$ and $\mathbf{1}-\mathrm{d}_{28}$. Indeed, we have confirmed that $1-\mathrm{d}_{28}$ undergoes peracid-mediated $\mathrm{B}-\mathrm{V}$ oxidation faster than nondeuterated $\mathbf{1}$, in accord with literature results (62), although a full kinetic analysis in the case of $\mathbf{1} / \mathbf{1}-\mathrm{d}_{28}$ was not possible due to partial overlap of ketone and lactone peaks under GC-MS conditions. Deuterium substitution is well known to affect drug pharmacokinetics [e.g., for drugs metabolized by aldehyde oxidase (65)], and can change many intermolecular interactions.

Critique of Current Theoretical Proposals. Turin's idea that electron transfer occurs at ORs and that these ORs can detect odorant vibrational frequencies has gained traction in recent years $(37$, 43-47). These theoretical works $(37,43,47)$ are in support of the vibration theory but remain largely tentative because they admittedly rely on unconfirmed assumptions, lacking experimental evidence, to make the proposal appear to be feasible. Electron transfer in biological environments is not uncommon. There is a substantial amount of literature reporting various experimental and theoretical studies. However, no evidence exists that GPCRs require electron transfer for their activation. The proposed mechanism (43) of delivering electrons to ORs is also too unreliable to set the stage for a failsafe mechanism of detecting odorants' vibrational frequencies. In addition, biological electron transfer processes are sensitive to chemical bonding characteristics, local molecular environments, and dynamic fluctuations, which can affect the transfer rate by orders of magnitudes. OR sites are floppy and open to numerous and diverse-sized odorants, and they are susceptible to these effects. Current theoretical proposals supporting the vibration theory, as summarized below, are oblivious to these complex issues.

Brookes et al. (43) proposed a model based on the standard spin-boson type of Hamiltonian for electron transfer (66), using Jortner's expression (67) of electron transfer rate for quantum vibrational modes. Two electron transfer times, $\tau_{0}$ for electron transfer without odorant and $\tau_{1}$ for electron transfer exciting one vibrational quantum of the odorant, were introduced. Assuming that all vibrational modes coupled to electron transfer are classical, except for the odorant oscillator, they obtain Marcus's expression (68) for $1 / \tau_{0}$ and Jortner's expression (67) for $1 / \tau_{1}(S I$ Appendix). Approximating the odorant oscillator as a classical point dipole, they estimated the Huang-Rhys factor of the odorant oscillator to be $\mathrm{S} \approx 0.01$. This value is very small, and would be difficult to detect unless high-quality samples and sensitive spectroscopic techniques are used. Brookes et al. (43) recognize this issue and propose that $(i)$ an OR site is finely tuned so that the energy difference between the electron donor and acceptor matches the vibrational quantum of the odorant oscillator, $E_{D}-E_{A}=\hbar \Omega$, and (ii) the reorganization energy of the protein environment is assumed to be very small, $c a$. $\lambda=30$ meV. Under these conditions, they conclude that electron transfer could detect odorant vibrational frequencies.

Although Brookes et al. (43) bring the vibration theory to a more concrete theoretical level, none of the key assumptions has supporting experimental evidence. Furthermore, their estimate for the reorganization energy of the electron transfer-coupled protein environment is unusually small even compared with other confirmed biological electron transfer processes (69). The reorganization energy for electron transfer in well-secured hydrophobic pockets of proteins can be small (70), but the assumed value (43) relies on old literature data (71), which is smaller by an order of magnitude than more recent estimates (72). Although the restriction on the reorganization energy can be relieved somewhat by modification of the resonance condition (SI Appendix), Solov'yov et al. (47) estimate that the reorganization energy needs to be smaller than $0.1 \mathrm{eV}$ for the vibration theory to be feasible. This value is still substantially smaller than commonly known values $(69,70)$. Clear experimental or computational evidence supporting such estimate is lacking.

Another fundamental issue with the proposed theoretical models $(43,47)$ is the neglect of quantum contributions from molecular vibrational modes other than those molecular vibrational modes of the odorant oscillator, leaving the window of vibrational frequencies open only for odorant molecules. This is tantamount to neglect of molecular-level structural information on OR sites available from homology models (47). The metalligand bonds and peptide bonds in the postulated electrondonor or acceptor sites could have similarly high-frequency modes with inelastic effects at least comparable to those high-frequency modes of odorants. Full consideration of such modes can easily alter the qualitative nature of electron transfer $(73,74)$ and could mask the vibrational frequencies of odorants, as illustrated in Fig. 5, making a cursory analysis of the vibration theory highly unreliable (a more detailed description is provided in SI Appendix).

The resulting values of detection efficiency $\tau_{0} / \tau_{1}$, plotted in Fig. 5, show that a modest amount of coupling to quantum vibrational modes of the environment could be sufficient to suppress the proposed odor detection mechanism of the vibration theory (43). In addition, considering the prevalence of $\mathrm{C}-\mathrm{H}$ bonds in protein environments, it is unclear how the effects of deuterating the odorants, as proposed by Gane et al. (14), can stand out even if the proposed electron transfer mechanism were

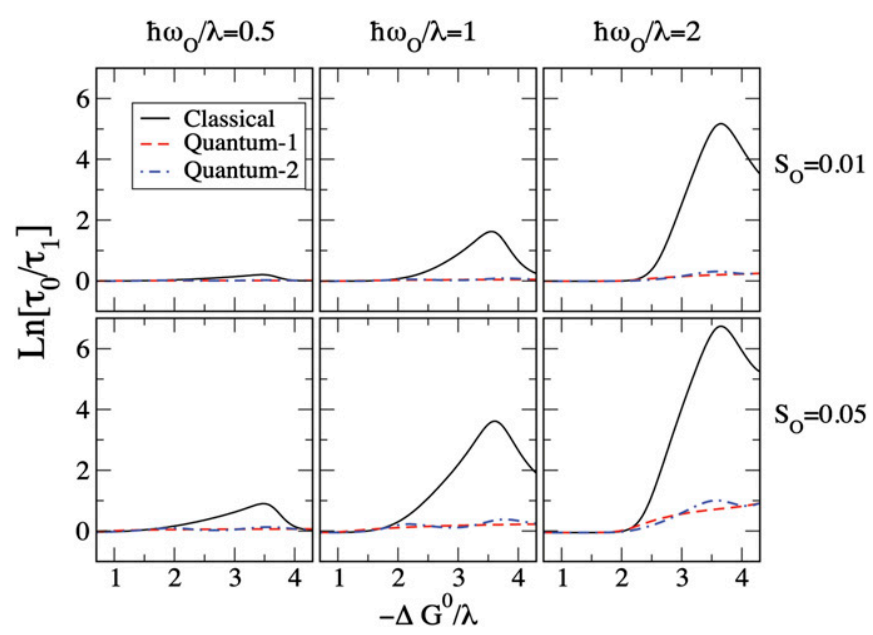

Fig. 5. Natural logarithms of the ratios of $\tau_{0} / \tau_{1}$ vs. $-\Delta G^{0} / \lambda$ (negative Gibbs free energy of reaction in the unit of reorganization energy) corresponding to the results in SI Appendix, Figs. S1.1-S1.3. The term "Classical" refers to the classical limit of Ohmic bath, SI Appendix, Eq. 18, with parameters for SI Appendix, Fig. S1.1. The term "Quantum-1" refers to the quantum regime of the Ohmic bath with parameters for SI Appendix, Fig. S1.2. The term "Quantum-2" refers to the case of classical Ohmic bath plus one quantum mode in the bath with parameters for SI Appendix, Fig. S1.3. Each column represents a different value of $\hbar \omega_{O} / \lambda$, where $\omega_{O}$ is the angular frequency of the odorant oscillator and $\lambda$ is the reorganization energy of the protein environments. $S_{O}=0.01$ (Upper) and $S_{O}=0.05$ (Lower), where $S_{O}$ is the Huang-Rhys factor for the odorant oscillator. 
true. Although Solov'yov et al. (47) made significant improvements to the work by Brookes et al. (43) by including the effects of more than one vibrational mode of odorants, calculating the Huang-Rhys factor in the presence of the field, and recognizing the importance of structural fitting of odorants, they also omitted consideration of nonodorant quantum vibrational modes. Thus, the issues raised above remain unresolved.

Bittner et al. (37) proposed a model where the electron transfer can occur only through the odorant as a bridge. The sensitivity to molecular vibration in this model also originates from resonance effects, assuming that internal modes of the odorant are excited impulsively during hole transfer from a donor site to acceptor site on the OR, along the direction of the gradient of the Born-Oppenheimer potential for its oxidized form. These assumptions lead to an interesting expression for the detection efficiency with direct correlation to IR signals and with some predictive capability. However, the final rate expression does not depend on the electronic energy of the odorant, which is at odds with most known multistate electron transfer processes (75). Most importantly, the model does not include the reorganization energy in the resonance condition, let alone the effects of quantum vibrational modes of donor and acceptor sites.

Other important issues that are not considered by all current theoretical proposals are the effects of disorder, dynamical fluctuations, and the sensitivity of electron couplings to bonding characteristics. For example, it is well established (75) that effective donor-acceptor electronic coupling is very sensitive to chemical characteristics and conformational details of the bridge (odorant) molecules [e.g., as shown by recent single-molecule conductance measurements, where substantial fluctuations of conductance were seen even when metallic electrodes are used under well-controlled bias potentials (76)]. Dynamical modulation of these electronic couplings is also very likely, resulting in fundamentally different kinetics $(77,78)$. Therefore, all of these factors can easily alter the electron transfer rate by orders of magnitude, becoming as important or more so, than the proposed Jortner-type vibronic effect (43). A theory that can genuinely support Turin's idea needs to demonstrate that electron transfer can indeed amplify small vibrational contributions of odorants despite all of these complicating effects typical of electron transfer in biological environments.

\section{Discussion}

In the absence of OR structural models, theoretical work is limited to the construction of phenomenological models consistent with available experimental observations. The principal experimental evidence supporting the vibrational theory has been the deuterium isotope effect at the perceptual (behavioral) level $(14,36,38)$. However, we find no experimental evidence supporting the theory at the molecular level. We focused on the functional analysis of a human OR tuned to the same musk compounds that were recently promoted as important experimental evidence, with the aim of specifically testing the electron tunneling mechanism at the receptor level. However, the experimental data reported in our study show a dramatic lack of correlation between OR-level signals and isotope effects over an extended set of 26 receptor/ligand pairs, with at least one deuterated counterpart to each of these ligands. In addition, we find that the assumptions of current theoretical models lack experimental support and do not necessarily fit into the general picture of typical electron transfer processes in biological environments. Thus, our combined experimental results and theoretical analysis present a comprehensive set of observations questioning the validity of the vibration theory as a plausible description of odor detection.

Gane et al. (14), finding that cyclopentadecanone (1) and $1-\mathrm{d}_{28}$ can be distinguished by human smell, speculated that "a small number of receptors, possibly just one, are involved in sensing musk odor," in accord with an earlier similar conclusion (55). A recent study (27) using heterologous cell assays and a c-fos induction assay in the olfactory bulb identified MOR215-1 as a strong musk-responding mouse OR. This study observes that " $6 \%$ of humans are muscone anosmic...; therefore, muscone may be recognized by only a small set of ORs, including OR5AN1 in humans, and genetic variation in these receptors may cause muscone anosmia” (27). McClintock et al. (54) identified five highly related ORs, including MOR215-1, that are likely to be activated by muscone in freely behaving mice, supporting multiple muscone receptors. Importantly, however, the only functional human counterpart of these ORs is OR5AN1. Here, we identify OR5AN1 through a thorough screening of the human OR repertoire using all four isotopomers of $\mathbf{1}$ and find that this OR responds similarly to these isotopomers. In summary, despite extensive screening, multiple research groups have identified only OR5AN1 as a human musk receptor. Nevertheless, failure to identify other human musk receptors in addition to OR5AN1 still leaves open the possibility that there are other human musk ORs. Future studies with genetic association with the OR5AN1 locus and/or development of OR5AN1-specific antagonist(s) could show whether OR5AN1 is the only OR that mediates behavioral responses to the musk compounds.

We supplement our study of the response of OR5AN1 to isotopomers with the analysis of the response of copperrequiring mouse receptor MOR244-3 to isotopomers of its most active ligands, as well as with the study of several other human and mouse receptors responding to cognate ligands. The consistent lack of difference found in the responses of all of these human and mouse receptors to isotopomers lessens our concern about possibly missing key receptors that are differentially responsive to isotopomers. Furthermore, we note it would be unusual for some, but not all, ORs strongly responsive to a particular ligand to demonstrate isotopomer discrimination.

In addition to OR5AN1, we describe here OR/ligand pairs of isotopomers of compounds 6-9. We found that none of the tested receptors exhibits different responses to isotopomers, although the IR spectra of the nondeuterated parents are strikingly different from the fully deuterated analogs (Fig. 2 and SI Appendix), and the inelastic electron-tunneling vibration frequencies would also be expected to differ significantly. Our experiments sought to examine the validity of the vibration theory at the receptor level by comparing the differential response to isotopomers, using a cell-based OR expression system, compared with differential responses found from animal behavioral studies or human odorant perception. So far, many studies have been able to correlate functional responses from intact neurons to functional responses of in vitro OR pharmacology. For example, by comparing the response profiles of several mouse ORs to cognate ligands using functional imaging of the olfactory bulb against heterologous ORs, Oka et al. (79) showed that ligand selectivity of the ORs is comparable, although the responses vary in efficacy. In addition, in vitro activity of human receptors in heterologous cells has been shown to predict human perception for several different ORs, also suggesting that the heterologous system at least partly mimics in vivo function $(80,81)$. Nonetheless, it should be noted that the current in vitro method is not without limitations. The experimental setup may lack the source of electrons assumed in the vibration theory. We cannot exclude the possibility that some ORs simply may not function in our system, thus reflecting only a fraction of OR responses that may be present at the perceptual level. One possibility is that the activation of certain ORs may lead to alternative signaling pathways and that our cAMP-based assay may not be able to detect such activation. In addition, the absence of a nasal mucosal environment prevents the evaluation of the significance of perireceptor events. 
With the limitations noted above, given the absence of an effect of deuteration on OR response, as demonstrated in the present work, and the lack of experimental evidence supporting the fundamental assumptions of current theoretical models of the vibration theory, we conclude that the perceived differences in smell and olfactory response are likely due to perireceptor processes or impurity of the tested odorants and not to inelastic electron tunneling assisted by vibrational modes.

\section{Conclusion}

Since Ogle's original proposal (1) for the vibration theory more than $140 \mathrm{y}$ ago, the idea has been embraced by Dyson $(4,5)$, Wright $(6-8)$, and Turin $(9,10)$. However, we find that it does not apply to the human musk receptor OR5AN1 or the mouse thiol receptor MOR244-3, as shown by the clear absence of isotope effects with deorphaned human and mouse ORs on exposure to the specific deuterated, ${ }^{13} \mathrm{C}$, and nonlabeled ligands for these ORs. Our testing included OR5AN1, which strongly responds identically to both muscone (4) and 4- $\mathrm{d}_{30}$. We also find that $4-\mathrm{d}_{30}$ lacks IR absorption in the 1,380 - to $1,550-\mathrm{cm}^{-1}$ range (Fig. 2), which is clearly at odds with the claims of Gane et al. (14) that a musk receptor "detects vibrations in the $1,380-1,550 \mathrm{~cm}^{-1}$ range," and that musk odor requires that "the molecule has intense bands in that region." Muscone- $\mathrm{d}_{30}$ has even more C-D bonds than found in ligands previously tested by Gane et al. (14), who claim that it is the number of hydrogen vibrational modes that is "essential for detecting the difference between isotopomers."

Our experimental results are consistent with the ability of mice (and other mammals) to discriminate between a large array of nonpheromonal chiral odorant enantiomeric pairs, as well as with the ability of mouse receptor MOR215-1 to discriminate $(R)$ - and $(S)$-muscone (27). Although it is known that the muscone enantiomers "differ from each other with regard to odor quality and the odor detection threshold in humans" (27), data are not yet available on the response of OR5AN1 to muscone enantiomers. We agree with the suggestion that the "muscone receptor is specific to $\mathrm{C} 15$ and $\mathrm{C} 16$ macrocyclic ketone compounds and that the ketone moiety may function as a hydrogen bond acceptor" (27). Although QM/MM and mutagenesis studies should elucidate the nature of the interaction of musks with OR5AN1, such a suggestion would be in accord with specific hydrogen bonding interactions as observed for the mouse eugenol receptor mOR-EG (82), as well as our observation that hydrocarbon analog cyclopentadecane (3), which lacks the carbonyl group of 1, is inactive toward OR5AN1 (SI Appendix, Fig. S3.2B).

Although some insect and human behavioral/psychophysical studies showed perceptual differences for isotopomers, perireceptor events or trace impurities may be sufficient to explain any isotope effect (2). Finally, with regard to the plausibility of the vibration theory, it has been argued that rather than being causal, any nonisotopic relationship between vibrational frequency and odor may come about indirectly as a consequence of

1. Ogle W (1870) Anosmia, or cases illustrating the physiology and pathology of the sense of smell. Med Chir Trans 53:263-290.

2. Sell CS (2014) Chemistry and the Sense of Smell (Wiley, Hoboken, NJ).

3. Moncrieff RW (1967) The Chemical Senses (Leonard Hill, London), 3rd Ed.

4. Dyson GM (1938) The scientific basis of odor. Chem Ind 57(28):647-651.

5. Dyson GM (1928) Some aspects of the vibration theory of odor. Perfumery and Essential Oil Record 19:456-459.

6. Wright RH, Reid C, Evans HGV (1956) Odor and molecular vibration. III. A new theory of olfactory stimulation. Chem Ind (37):973-977.

7. Wright RH (1977) Odor and molecular vibration: Neural coding of olfactory information. J Theor Biol 64(3):473-502.

8. Wright RH (1961) Odour and molecular vibration. Nature 190(4781):1101-1102.

9. Turin L (1996) A spectroscopic mechanism for primary olfactory reception. Chem Senses 21(6):773-791.

10. Turin $L$ (1997) The nose as spectroscopist. Chem Ind (21):866-870.

11. Burr C (2002) The Emperor of Scent: A Story of Perfume, Obsession, and the Last Mystery of the Senses (Random House, New York).

12. Gilbert AN (2003) The emperor's new theory. Nat Neurosci 6(4):335. "similar molecules having similar properties" (83) and because "the vibration spectrum of a molecule reflects its structure" (84). Our findings that the vibration theory is not supported by rigorous analysis of the response of OR5AN1 to diverse isotopomers reinforce Sell's recommendation (85) that those individuals "wishing to study the nature of odorant-receptor recognition should use receptor activation rather than odor as input data."

\section{Materials and Methods}

Chemicals. All odorants were purchased from Sigma-Aldrich, J\&K, or Chemsky, or were synthesized in-house. Deuterium incorporation into compounds $\mathbf{1 - 4}, \mathbf{8}$, and $\mathbf{9}$ was accomplished by methods reported in the literature from undeuterated or deuterated commercially available starting materials, as described in SI Appendix, with full characterization of all compounds, following purification by chromatography and recrystallization to a constant melting point (when possible), by ${ }^{1} \mathrm{H}$ and ${ }^{13} \mathrm{C}$ NMR, IR spectroscopy, and GC-MS. Spectra and GC-MS traces are included in SI Appendix. The chemicals were dissolved in DMSO or ethanol and diluted further into working concentrations before experiments.

Heterologous Expression of ORs. A HEK 293T-derived Hana3A cell line was grown in Minimum Essential Medium (HyClone) containing 10\% (vol/vol) FBS at $37{ }^{\circ} \mathrm{C}$ with $5 \%$ (vol/vol) $\mathrm{CO}_{2}$. Lipofectamine 2000 (Invitrogen) was used for transfection. Luciferase assays were performed as previously described. After 18-24 h, OR, the accessory OR protein, mRTP1S, and constructs for firefly luciferase and Renilla luciferase expression were transfected into cells. Twenty-four hours after transfection, the cells were stimulated with odorants [plus $30 \mu \mathrm{M} \mathrm{Cu}{ }^{2+}$ ions when the ligands were MTMT-, bis(methylthiomethyl) disulfide, and dimethyl sulfide-dissolved in CD293 (Invitrogen)]. We used the Dual-Glo Luciferase Assay System (Promega) and followed the manufacturer's instructions for measuring chemiluminescence.

Statistical Analyses. One-way ANOVA or an unpaired Student's $t$ test was used to compare the $95 \%$ confidence interval $\log _{50}$ values among isotopomers for each receptor/odorant pair in Figs. 3 and 4 . The level of significance was $* P<0.05$. An $F$ test was used to compare the best-fit values of $E_{50}$, Hill slope, and top of the dose-response curves between the original hydrogenated odorant and its isotopomers in Figs. 3 and 4 and SI Appendix, Fig. S3.2. Bonferroni correction was applied to the $F$ tests to account for multiple comparisons. The level of significance was ${ }^{*} P<0.00076$ before correction and $* P<0.05$ after correction.

ACKNOWLEDGMENTS. We thank Marshall Newton, Leslie Vosshall, Avery Gilbert, and Andreas Keller for their valuable comments on the manuscript. The authors acknowledge support from the National Science Foundation (Grant CHE-1265679 to E.B. and Grant CHE-1362926 to S.J.); the National Science Foundation Faculty Early Career Development Program (CAREER Grant CHE-0846899 to S.J. and Grant CHE-1213742 to V.S.B.); the Chinese Academy of Sciences for a Visiting Professorship for Senior International Scientists (to E.B.); the Camille Dreyfus Teacher Scholar Award (to S.J.); the Program for Innovative Research Team of Shanghai Municipal Education Commission (H.Z.); the Shanghai Eastern Scholar Program (Grant J50201 to H.Z.); the National Basic Research Program of China (Grant 2012CB910401 to H.Z.); a Computational Materials and Chemical Sciences project under Contract DE-AC02-98CH10886 with the US Department of Energy and supported by its Division of Chemical Sciences, Geosciences, and Biosciences, Office of Basic Energy Sciences (to M.Z.E.); and the NIH (Grants DC005782 and DC012095 to H.M.)

13. Wolf EL (1985) Principles of Electronic Tunneling Spectroscopy (Oxford Univ Press, New York).

14. Gane S, et al. (2013) Molecular vibration-sensing component in human olfaction. PLoS ONE 8(1):e55780.

15. Duan $X$, et al. (2012) Crucial role of copper in detection of metal-coordinating odorants. Proc Natl Acad Sci USA 109(9):3492-3497.

16. Sekharan S, et al. (2014) QM/MM model of the mouse olfactory receptor MOR244-3 validated by site-directed mutagenesis experiments. Biophys J 107(5):L5-L8 .

17. Franco MI, Turin L, Mershin A, Skoulakis EMC (2011) Reply to Hettinger: Olfaction is a physical and a chemical sense in Drosophila. Proc Natl Acad Sci USA 108(31):E350 (lett).

18. Hettinger TP (2011) Olfaction is a chemical sense, not a spectral sense. Proc Nat/ Acad Sci USA 108(31): E349 (lett).

19. Wade D (1999) Deuterium isotope effects on noncovalent interactions between molecules. Chem Biol Interact 117(3):191-217.

20. Schramm VL (2007) Binding isotope effects: Boon and bane. Curr Opin Chem Biol 11(5):529-536.

21. Pauling L, Delbrück M (1940) The nature of the intermolecular forces operative in biological processes. Science 92(2378):77-79. 
22. Turin L (2005) Rational odorant design. Chemistry and Technology of Flavors and Fragrances, ed Rowe DJ (Blackwell, Oxford), pp 261-273.

23. Rizvanovic A, Amundin M, Laska M (2013) Olfactory discrimination ability of Asian elephants (Elephas maximus) for structurally related odorants. Chem Senses 38(2): 107-118.

24. Laska M, Shepherd GM (2007) Olfactory discrimination ability of CD-1 mice for a large array of enantiomers. Neuroscience 144(1):295-301.

25. Rubin BD, Katz LC (2001) Spatial coding of enantiomers in the rat olfactory bulb. Nat Neurosci 4(4):355-356.

26. Saito H, Chi Q, Zhuang H, Matsunami H, Mainland JD (2009) Odor coding by a Mammalian receptor repertoire. Sci Signal 2(60):ra9.

27. Shirasu M, et al. (2014) Olfactory receptor and neural pathway responsible for highly selective sensing of musk odors. Neuron 81(1):165-178.

28. Axel R (2005) Scents and sensibility: A molecular logic of olfactory perception (Nobe lecture). Angew Chem Int Ed Engl 44(38):6110-6127.

29. Buck LB (2005) Unraveling the sense of smell (Nobel lecture). Angew Chem Int Ed Engl 44(38):6128-6140.

30. Haffenden LJW, Yaylayan VA, Fortin J (2001) Investigation of vibrational theory of olfaction with variously labelled benzaldehydes. Food Chem 73(1):67-72.

31. Keller A, Vosshall LB (2004) A psychophysical test of the vibration theory of olfaction. Nat Neurosci 7(4):337-338.

32. Nagashima A, Touhara K (2010) Enzymatic conversion of odorants in nasal mucus affects olfactory glomerular activation patterns and odor perception. $J$ Neurosci 30(48):16391-16398

33. Raju VS, Sharma PK, Banerji KK (2000) Kinetics and mechanism of the oxidation of substituted benzaldehydes by benzyltrimethylammonium chlorobromate. J Org Chem 65(11):3322-3325.

34. Seok WK, Meyer TJ (2005) Mechanism of oxidation of benzaldehyde by polypyridyl oxo complexes of Ru(IV). Inorg Chem 44(11):3931-3941.

35. Turin L, Yoshii F (2003) Structure-odor relations: A modern perspective. Handbook of Olfaction and Gustation, ed Doty RL (Marcel Dekker, New York), pp 275-294.

36. Franco MI, Turin L, Mershin A, Skoulakis EM (2011) Molecular vibration-sensing component in Drosophila melanogaster olfaction. Proc Natl Acad Sci USA 108(9): 3797-3802.

37. Bittner ER, Madalan A, Czader A, Roman G (2012) Quantum origins of molecular recognition and olfaction in Drosophila. J Chem Phys 137(22):22A551.

38. Gronenberg W, et al. (2014) Honeybees (Apis mellifera) learn to discriminate the smell of organic compounds from their respective deuterated isotopomers. Proc Biol Sci 281(1778):20133089.

39. Doolittle RE, Beroza M, Keiser I, Schneider EL (1968) Deuteration of the melon fly attractant, cue-lure, and its effect on olfactory response and infra-red absorption. $J$ Insect Physiol 14(12):1697-1712.

40. Blum MS, Doolittle RE, Beroza M (1971) Alarm pheromones: Utilization in evaluation of olfactory theories. J Insect Physiol 17(12):2351-2361.

41. Barker RJ, Berdel RL, Waller GD (1973) The molecular basis for scent discrimination: Response to nitrobenzene-d5 of honey bees (Apis mellifera L.) conditioned with nitrobenzene. Experientia 29(4):418-419.

42. Klika KD (2013) The potential of ${ }^{13} \mathrm{C}$ isotopomers as a test for the vibrational theory of olfactory sense recognition. ISRN Org Chem 2013:515810.

43. Brookes JC, Hartoutsiou F, Horsfield AP, Stoneham AM (2007) Could humans recognize odor by phonon assisted tunneling? Phys Rev Lett 98(3):038101.

44. Brookes JC (2010) Science is perception: What can our sense of smell tell us about ourselves and the world around us? Philos Trans A Math Phys Eng Sci 368(1924): $3491-3502$.

45. Brookes JC (2011) Olfaction: The physics of how smell works? Contemp Phys 52(5): 385-402.

46. Brookes JC, Horsfield AP, Stoneham AM (2012) The swipe card model of odorant recognition. Sensors (Basel) 12(11):15709-15749.

47. Solov'yov IA, Chang PY, Schulten K (2012) Vibrationally assisted electron transfer mechanism of olfaction: Myth or reality? Phys Chem Chem Phys 14(40):13861-13871.

48. Winkler JR, Gray HB (2014) Long-range electron tunneling. J Am Chem Soc 136(8): 2930-2939.

49. Maegawa T, et al. (2008) Mild and efficient H/D exchange of alkanes based on C-H activation catalyzed by rhodium on charcoal. Angew Chem Int Ed Engl 47(29): 5394-5397.

50. Harding KE, May LM, Dick KF (1975) Selective oxidation of allylic alcohols with chromic acid. J Org Chem 40(11):1664-1665.

51. Wesslen B (1968) Aldol reactions of formaldehyde in non-aqueous media. 4. Mech anism of acid-catalyzed reaction of 2-butanone with formaldehyde. Acta Chem Scand 22(7):2085-2110

52. Saito H, Kubota M, Roberts RW, Chi Q, Matsunami H (2004) RTP family members induce functional expression of mammalian odorant receptors. Cell 119(5):679-691.
53. Zhuang H, Matsunami H (2008) Evaluating cell-surface expression and measuring activation of mammalian odorant receptors in heterologous cells. Nat Protoc 3(9): $1402-1413$.

54. McClintock TS, et al. (2014) In vivo identification of eugenol-responsive and musconeresponsive mouse odorant receptors. J Neurosci 34(47):15669-15678.

55. Nara K, Saraiva LR, Ye X, Buck LB (2011) A large-scale analysis of odor coding in the olfactory epithelium. J Neurosci 31(25):9179-9191.

56. Morton TH (2000) Archiving odors. Of Molecules and Mind, eds Bhushan $\mathrm{N}$, Rosenfeld S (Oxford Univ Press, Oxford), pp 251-272.

57. Schilling B, Kaiser R, Natsch A, Gautschi M (2010) Investigation of odors in the fragrance industry. Chemoecology 20:135-147.

58. Mozell MM (1970) Evidence for a chromatographic model of olfaction. J Gen Physiol 56(1):46-63.

59. Hahn I, Scherer PW, Mozell MM (1994) A mass transport model of olfaction. J Theor Biol 167(2):115-128.

60. Wilkes FJ, Laing DG, Hutchinson I, Jinks AL, Monteleone E (2009) Temporal processing of olfactory stimuli during retronasal perception. Behav Brain Res 200(1):68-75.

61. Turowski M, et al. (2003) Deuterium isotope effects on hydrophobic interactions: The importance of dispersion interactions in the hydrophobic phase. J Am Chem Soc 125(45):13836-13849.

62. Renz M, Meunier B (1999) 100 years of Baeyer-Villager oxidations. European J Org Chem 1999(4):737-750.

63. Zhuo X et al. (1999) Biotransformation of coumarin by rodent and human cytochromes P-450: Metabolic basis of tissue-selective toxicity in olfactory mucosa of rats and mice. J Pharmacol Exp Ther 288(2):463-471.

64. Thiebaud $N$, et al. (2013) Odorant metabolism catalyzed by olfactory mucosal en zymes influences peripheral olfactory responses in rats. PLOS ONE 8(3):e59547.

65. Sharma R, et al. (2012) Deuterium isotope effects on drug pharmacokinetics. I. System-dependent effects of specific deuteration with aldehyde oxidase cleared drugs. Drug Metab Dispos 40(3):625-634.

66. Leggett AJ, et al. (1987) Dynamics of the dissipative two-state system. Rev Mod Phys 59(1):1-85.

67. Jortner J (1976) Temperature-dependent activation-energy for electron-transfer between biological molecules. J Chem Phys 64(12):4860-4867.

68. Marcus RA (1965) On theory of electron-transfer reactions. 6. Unified treatment for homogeneous and electrode reactions. J Chem Phys 43(2):679-701.

69. Moser CC, Keske JM, Warncke K, Farid RS, Dutton PL (1992) Nature of biological electron transfer. Nature 355(6363):796-802

70. Gray HB, Winkler JR (1996) Electron transfer in proteins. Annu Rev Biochem 65 537-561.

71. Jia $Y$, et al. (1993) Primary charge separation in mutant reaction centers of Rhodo bacter capsulatus. J Phys Chem 97(50):13180-13191.

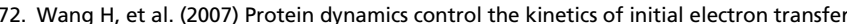
in photosynthesis. Science 316(5825):747-750.

73. Ulstrup J, Jortner J (1975) Effect of intramolecular quantum modes on free-energy relationships for electron-transfer reactions. J Chem Phys 63(10):4358-4368.

74. Jang S, Newton MD (2006) Closed-form expressions of quantum electron transfer rate based on the stationary-phase approximation. J Phys Chem B 110(38):18996-19003.

75. Jortner J (1999) Electron Transfer-From Isolated Molecules to Biomolecules, ed Bixon M (Wiley, Hoboken, NJ), Vol 106.

76. Venkataraman L, Klare JE, Nuckolls C, Hybertsen MS, Steigerwald ML (2006) Dependence of single-molecule junction conductance on molecular conformation. $\mathrm{Na}$ ture 442(7105):904-907.

77. Medvedev ES, Stuchebrukhov AA (1997) Inelastic tunneling in long-distance bi ological electron transfer reactions. J Chem Phys 107(10):3821-3831.

78. Jang S, Newton MD (2005) Theory of torsional non-Condon electron transfer: A generalized spin-boson Hamiltonian and its nonadiabatic limit solution. $J$ Chem Phys 122(2):024501.

79. Oka $Y$, et al. (2006) Odorant receptor map in the mouse olfactory bulb: In vivo sensitivity and specificity of receptor-defined glomeruli. Neuron 52(5):857-869.

80. Keller A, Zhuang H, Chi Q, Vosshall LB, Matsunami H (2007) Genetic variation in a human odorant receptor alters odour perception. Nature 449(7161):468-472.

81. Menashe I, et al. (2007) Genetic elucidation of human hyperosmia to isovaleric acid. PLOS Biol 5(11):e284.

82. Baud O, et al. (2011) The mouse eugenol odorant receptor: Structural and functional plasticity of a broadly tuned odorant binding pocket. Biochemistry 50(5):843-853.

83. Takane SY, Mitchell JB (2004) A structure-odour relationship study using EVA descriptors and hierarchical clustering. Org Biomol Chem 2(22):3250-3255.

84. Gabler S, Soelter J, Hussain T, Sachse S, Schmuker M (2013) Physicochemical vs. vibrational descriptors for prediction of odor receptor responses. Mol Inform 32(9-10): 855-865.

85. Triller A, et al. (2008) Odorant-receptor interactions and odor percept: A chemical perspective. Chem Biodivers 5(6):862-886. 